\title{
LOCAL BOUNDARY CONDITIONS IN QUANTUM SUPERGRAVITY
}

\section{Giampiero Esposito}

\author{
Istituto Nazionale di Fisica Nucleare, Sezione di Napoli \\ Mostra d'Oltremare Padiglione 20, 80125 Napoli, Italy; \\ Dipartimento di Scienze Fisiche \\ Mostra d'Oltremare Padiglione 19, 80125 Napoli, Italy.
}

\begin{abstract}
When quantum supergravity is studied on manifolds with boundary, one may consider local boundary conditions which fix on the initial surface the whole primed part of tangential components of gravitino perturbations, and fix on the final surface the whole unprimed part of tangential components of gravitino perturbations. This paper studies such local boundary conditions in a flat Euclidean background bounded by two concentric 3 -spheres. It is shown that, as far as transverse-traceless perturbations are concerned, the resulting contribution to $\zeta(0)$ vanishes when such boundary data are set to zero, exactly as in the case when non-local boundary conditions of the spectral type are imposed. These properties may be used to show that one-loop finiteness of massless supergravity models is only achieved when two boundary 3-surfaces occur, and there is no exact cancellation of the contributions of gauge and ghost modes in the Faddeev-Popov path integral. In these particular cases, which rely on the use of covariant gauge-averaging functionals, pure gravity is one-loop finite as well.
\end{abstract}




\section{Local Boundary Conditions in Quantum Supergravity}

The problem of a consistent formulation of quantum supergravity on manifolds with boundary is still receiving careful consideration in the current literature [1-4]. In particular, many efforts have been produced to understand whether simple supergravity is one-loop finite (or even finite to all orders of perturbation theory [3]) in the presence of boundaries. In the analysis of such an issue, the first problem consists, of course, in a careful choice of boundary conditions. For massless gravitino potentials, which are the object of our investigation, these may be non-local of the spectral type [5] or local [1-4].

In the former case the idea is to fix at the boundary half of the gravitino potential. On the final surface $\Sigma_{F}$ one can fix those perturbative modes which multiply harmonics having positive eigenvalues of the intrinsic three-dimensional Dirac operator $\mathcal{D}$ of the boundary. On the initial surface one can instead fix those gravitino modes which multiply harmonics having negative eigenvalues of the intrinsic three-dimensional Dirac operator of the boundary. What is non-local in this procedure is the separation of the spectrum of a first-order elliptic operator (our $\mathcal{D}$ ) into a positive and a negative part. This leads to a sort of positive- and negative-frequency split which is typical for scattering problems [3], but may also be applied to the analysis of quantum amplitudes in finite regions [4].

Our paper deals instead with the latter choice, i.e. local boundary conditions for quantum supergravity. By this one usually means a formulation where complementary projection operators act on gravitational and spin- $\frac{3}{2}$ perturbations. Local boundary conditions of this type were investigated in Refs. [6,7], and then applied to quantum cosmological backgrounds in Refs. [1,4,8-10].

More recently, another choice of local boundary conditions for gravitino perturbations has been considered in Ref. [3]. Using two-component spinor notation, and referring the reader to Refs. [3,11] for notation and background material, we here represent the spin- $\frac{3}{2}$ potential by a pair of independent spinor-valued one-forms $\left(\psi_{\mu}^{A}, \widetilde{\psi}_{\mu}^{A^{\prime}}\right)$ in a Riemannian 4manifold which is taken to be flat Euclidean 4-space bounded by two concentric 3-spheres $[2,4]$. Denoting by $S_{I}$ and $S_{F}$ the boundary 3 -spheres, with radii $a$ and $b$ respectively (here $b>a)$, the local boundary conditions proposed in Ref. [3] read in our case $(i=1,2,3)$

$$
\left[\widetilde{\psi}_{i}^{A^{\prime}}\right]_{S_{I}}=F_{i}^{A^{\prime}}
$$


Local Boundary Conditions in Quantum Supergravity

$$
\left[\psi_{i}^{A}\right]_{S_{F}}=H_{i}^{A}
$$

where $F_{i}^{A^{\prime}}$ and $H_{i}^{A}$ are boundary data which may or may not satisfy the classical constraint equations [3]. With the choice (1) and (2), the whole primed part of the tangential components of the spin- $\frac{3}{2}$ potential is fixed on $S_{I}$, and the whole unprimed part of the tangential components of the spin- $\frac{3}{2}$ potential is fixed on $S_{F}$.

In a Hamiltonian analysis, $\psi_{0}^{A}$ and $\widetilde{\psi}_{0}^{A^{\prime}}$ are Lagrange multipliers, and hence boundary conditions for them look un-natural in a one-loop calculation [3], especially if one is interested in reduction to transverse-traceless (TT) gravitino modes (usually regarded as the physical part of gravitinos). In a covariant path-integral analysis, however, one cannot disregard the issue of boundary conditions on normal components of gravitinos. We shall thus post-pone the discussion of this point, and we will focus on the TT sector of the boundary conditions (1) and (2).

With the notation of Ref. [1], the expansion in harmonics on 3-spheres of the TT part of tangential components of spin- $\frac{3}{2}$ perturbations reads

$$
\begin{gathered}
\psi_{i}^{A}=\frac{\tau^{-\frac{3}{2}}}{2 \pi} \sum_{n=0}^{\infty} \sum_{p, q=1}^{(n+1)(n+4)} \alpha_{n}^{p q}\left[m_{n p}(\tau) \beta^{n q A C C^{\prime}}+\widetilde{r}_{n p}(\tau) \bar{\mu}^{n q A C C^{\prime}}\right] e_{C C^{\prime} i} \\
\widetilde{\psi}_{i}^{A^{\prime}}=\frac{\tau^{-\frac{3}{2}}}{2 \pi} \sum_{n=0}^{\infty} \sum_{p, q=1}^{(n+1)(n+4)} \alpha_{n}^{p q}\left[\widetilde{m}_{n p}(\tau) \bar{\beta}^{n q A^{\prime} C^{\prime} C}+r_{n p}(\tau) \mu^{n q A^{\prime} C^{\prime} C}\right] e_{C C^{\prime} i},
\end{gathered}
$$

where $\beta^{n q A C C^{\prime}} \equiv-\rho^{n q(A C D)} n_{D} C^{\prime}$ and $\mu^{n q A^{\prime} C^{\prime} C} \equiv-\sigma^{n q\left(A^{\prime} C^{\prime} D^{\prime}\right)} n_{D^{\prime}}$. Of course, round brackets denote complete symmetrization over spinor indices, and $n_{D^{\prime}}^{C}$ is obtained from the Euclidean normal as $n_{D^{\prime}}^{C}=i_{e} n_{D^{\prime}}^{C}$. Variation of the TT gravitino action yields, for all integer $n \geq 0$, the following eigenvalue equations for gravitino modes:

$$
\begin{gathered}
\left(\frac{d}{d \tau}-\frac{(n+5 / 2)}{\tau}\right) x_{n p}=E_{n p} \widetilde{x}_{n p}, \\
\left(-\frac{d}{d \tau}-\frac{(n+5 / 2)}{\tau}\right) \widetilde{x}_{n p}=E_{n p} x_{n p},
\end{gathered}
$$




\section{Local Boundary Conditions in Quantum Supergravity}

where $x_{n p}=m_{n p}$ and $\widetilde{x}_{n p}=\widetilde{m}_{n p}$, or $x_{n p}=r_{n p}$ and $\widetilde{x}_{n p}=\widetilde{r}_{n p}$. The Eqs. (5) and (6) lead to the following basis functions in terms of modified Bessel functions (hereafter $M \equiv E_{n p}$ for simplicity of notation, while $\beta_{1, n}$ and $\beta_{2, n}$ are some constants):

$$
\begin{gathered}
m_{n p}(\tau)=\beta_{1, n} \sqrt{\tau} I_{n+2}(M \tau)+\beta_{2, n} \sqrt{\tau} K_{n+2}(M \tau), \\
\widetilde{m}_{n p}(\tau)=\beta_{1, n} \sqrt{\tau} I_{n+3}(M \tau)-\beta_{2, n} \sqrt{\tau} K_{n+3}(M \tau), \\
r_{n p}(\tau)=\beta_{1, n} \sqrt{\tau} I_{n+2}(M \tau)+\beta_{2, n} \sqrt{\tau} K_{n+2}(M \tau), \\
\widetilde{r}_{n p}(\tau)=\beta_{1, n} \sqrt{\tau} I_{n+3}(M \tau)-\beta_{2, n} \sqrt{\tau} K_{n+3}(M \tau) .
\end{gathered}
$$

By virtue of (1) and (2), these modes obey the boundary conditions

$$
\begin{gathered}
\tilde{m}_{n p}(a)=A_{n}, \\
r_{n p}(a)=A_{n}, \\
m_{n p}(b)=B_{n}, \\
\widetilde{r}_{n p}(b)=B_{n},
\end{gathered}
$$

where $A_{n}$ and $B_{n}$ are constants resulting from the boundary data $F_{i}^{A^{\prime}}$ and $H_{i}^{A}$ respectively. The boundary conditions (11) and (12) lead therefore, if $A_{n}=B_{n}=0, \forall n$, to the eigenvalue condition

$$
I_{n+3}(M r) K_{n+2}(M r)+I_{n+2}(M r) K_{n+3}(M r)=0,
$$

where $r=a$ or $b$.

We can now apply $\zeta$-function regularization to evaluate the resulting TT contribution to the one-loop divergence, following the algorithm developed in Ref. [12]. The basic steps are as follows. Let us denote by $f_{l}$ the function occurring in the equation obeyed by the eigenvalues by virtue of boundary conditions, after taking out fake roots (e.g. $x=0$ is a fake root of order $n$ of the Bessel function $\left.I_{n}\right)$. Let $d(l)$ be the degeneracy of the eigenvalues parametrized by the integer $l$. One can then define the function

$$
I\left(M^{2}, s\right) \equiv \sum_{l=l_{0}}^{\infty} d(l) l^{-2 s} \log f_{l}\left(M^{2}\right)
$$




\section{Local Boundary Conditions in Quantum Supergravity}

Such a function admits an analytic continuation to the complex-s plane as a meromorphic function with a simple pole at $s=0$, in the form

$$
" I\left(M^{2}, s\right) "=\frac{I_{\text {pole }}\left(M^{2}\right)}{s}+I^{R}\left(M^{2}\right)+\mathrm{O}(s) .
$$

The function $I_{\text {pole }}\left(M^{2}\right)$ is the residue at $s=0$, and makes it possible to obtain the $\zeta(0)$ value as

$$
\zeta(0)=I_{\log }+I_{\text {pole }}\left(M^{2}=\infty\right)-I_{\text {pole }}\left(M^{2}=0\right),
$$

where $I_{\log }$ is the coefficient of the $\log M$ term in $I^{R}$ as $M \rightarrow \infty$. Moreover, $I_{\text {pole }}(\infty)$ coincides with the coefficient of $\frac{1}{l}$ in the expansion as $l \rightarrow \infty$ of $\frac{1}{2} d(l) \log \left[\rho_{\infty}(l)\right]$, where $\rho_{\infty}(l)$ is the $l$-dependent term in the eigenvalue condition as $M \rightarrow \infty$ and $l \rightarrow \infty$. The $I_{\text {pole }}(0)$ value is instead obtained as the coefficient of $\frac{1}{l}$ in the expansion as $l \rightarrow \infty$ of $\frac{1}{2} d(l) \log \left[\rho_{0}(l)\right]$, where $\rho_{0}(l)$ is the $l$-dependent term in the eigenvalue condition as $M \rightarrow 0$ and $l \rightarrow \infty$.

In our problem, using the limiting form of Bessel functions when the argument tends to zero, one finds that the left-hand side of (13) is proportional to $M^{-1}$ as $M \rightarrow 0$. Hence one has to multiply by $M$ to get rid of fake roots. Moreover, in the uniform asymptotic expansion of Bessel functions as $M \rightarrow \infty$ and $n \rightarrow \infty$, both $I$ and $K$ functions contribute a $\frac{1}{\sqrt{M}}$ factor. These properties imply that $I_{\log }$ vanishes (hereafter $l \equiv n+1$ ):

$$
I_{\log }=\frac{1}{2} \sum_{l=1}^{\infty} 2 l(l+3)(1-1 / 2-1 / 2)=0 .
$$

Moreover, $I_{\text {pole }}(\infty)$ vanishes since there is no $l$-dependent coefficient in the uniform asymptotic expansion of (13) as $M \rightarrow \infty$ and $l \rightarrow \infty$. Last, $I_{\text {pole }}(0)$ vanishes as well, since the limiting form of (13) as $M \rightarrow 0$ and $l \rightarrow \infty$ is $\frac{1}{r} M^{-1}$. One thus finds for gravitinos

$$
\zeta_{T T}(0)=0 \text {. }
$$

It is now clear that local boundary conditions for $\psi_{0}^{A}$ and $\widetilde{\psi}_{0}^{A^{\prime}}$ along the same lines of (1) and (2), i.e. (here $\kappa^{A^{\prime}}$ and $\mu^{A}$ are some boundary data)

$$
\left[\widetilde{\psi}_{0}^{A^{\prime}}\right]_{S_{I}}=\kappa^{A^{\prime}}
$$


Local Boundary Conditions in Quantum Supergravity

$$
\left[\psi_{0}^{A}\right]_{S_{F}}=\mu^{A}
$$

give again a vanishing contribution to $\zeta(0)$ in this two-boundary problem if $\kappa^{A^{\prime}}$ and $\mu^{A}$ are set to zero, since the resulting eigenvalue condition is analogous to (13) with $n$ replaced by $n-1$.

A suitable set of local boundary conditions on metric perturbations $h_{\mu \nu}$ are the ones considered by Luckock, Moss and Poletti [6-8]. In our problem, denoting by $g_{\mu \nu}$ the background 4-metric, they read $[9,10]$

$$
\begin{gathered}
{\left[h_{i j}\right]_{\partial M}=0,} \\
{\left[\frac{\partial h_{00}}{\partial \tau}+\frac{6}{\tau} h_{00}-\frac{\partial}{\partial \tau}\left(g^{i j} h_{i j}\right)\right]_{\partial M}=0,} \\
{\left[h_{0 i}\right]_{\partial M}=0,}
\end{gathered}
$$

while the ghost 1-form is subject to the mixed boundary conditions [10]

$$
\begin{gathered}
{\left[\varphi_{0}\right]_{\partial M}=0} \\
{\left[\frac{\partial \varphi_{i}}{\partial \tau}-\frac{2}{\tau} \varphi_{i}\right]_{\partial M}=0 .}
\end{gathered}
$$

As shown in Ref. [9], graviton TT modes contribute

$$
\zeta_{T T}(0)=-5
$$

while, using a covariant gauge-averaging functional of the de Donder type, gauge and ghost modes contribute [9]

$$
\zeta(0)_{\text {gauge and ghost }}=5 .
$$

One thus finds that the one-loop path integral for the gravitational sector, including TT, gauge and ghost modes, gives a vanishing contribution to the one-loop divergence. 


\section{Local Boundary Conditions in Quantum Supergravity}

What is left are gauge and ghost modes for gravitino perturbations. In general, their separate values depend on the gauge-averaging functional being used (only the full oneloop divergence should be gauge-independent [13]). However, on general ground, since in our flat Euclidean background all possible contributions to $\zeta(0)$ involve surface integrals of terms like $[3,14]$

$$
\operatorname{Tr}\left(K^{3}\right),(\operatorname{Tr} K)\left(\operatorname{Tr} K^{2}\right),(\operatorname{Tr} K)^{3}
$$

$K$ denoting the extrinsic-curvature tensor of the boundary, any dependence on the 3spheres radii $a$ and $b$ disappears after integration over $\partial M$. Moreover, if the formalism is gauge-independent ax expected, the one-loop result can only coincide with the one found in Sec. V of Ref. [4] in the axial gauge:

$$
\zeta_{\frac{3}{2}}(0)=0
$$

The following concluding remarks are now in order:

(i) The one-loop finiteness suggested by our analysis does not seem to contradict the results of Ref. [4]. What is shown in Ref. [4] is instead that, when flat Euclidean 4-space is bounded by only one 3-sphere, simple supergravity fails to be one-loop finite (either spectral boundary conditions with non-covariant gauge, or local boundary conditions in covariant gauge). This is not our background. Moreover, the two-boundary problem of Ref. [4] was studied in a non-covariant gauge of the axial type, when the Faddeev-Popov path integral is supplemented by the integrability condition for the eigenvalue equations on graviton and gravitino perturbations. This quantization was found to pick out TT modes only [4], but differs from the scheme proposed in our paper.

(ii) The result (28) is crucial for one-loop finiteness to hold (when combined with (26) and (27)), and its explicit proof has not yet been achieved.

(iii) As a further check of one-loop finiteness (or of its lack), one should now perform two-boundary calculations of $\zeta(0)$ when Luckock-Moss-Poletti local boundary conditions are imposed on gravitino perturbations. 


\section{Local Boundary Conditions in Quantum Supergravity}

(iv) Yet another check might be obtained by combining Barvinsky boundary conditions for pure gravity $[10,15]$ in the de Donder gauge (these are completely invariant under infinitesimal diffeomorphisms) with local or non-local boundary conditions for gravitinos in covariant gauges [13].

(v) The calculations performed in Refs. $[4,9,10,16]$ and in our paper show that, when cancellation of the effects of gauge and ghost modes is achieved, only the effects of TT modes survive, and hence both pure gravity and simple supergravity are not even one-loop finite. By contrast, if the effects of gauge and ghost modes do not cancel each other exactly (e.g. by using covariant gauge-averaging functionals in the Faddeev-Popov path integral), then both pure gravity and simple supergravity may turn out to be one-loop finite in the presence of two bounding 3-spheres.

(vi) A deep problem is the relation between the Hamiltonian analysis of Ref. [3], where auxiliary fields play an important role but ghost fields are not studied, and the pathintegral approach of Ref. [4], where ghost fields are analyzed in detail but auxiliary fields are not found to affect the one-loop calculation.

All this adds evidence in favour of quantum cosmology being able to lead to new perspectives in Euclidean quantum gravity and quantum supergravity.

I am much indebted to A. Yu. Kamenshchik for scientific collaboration on $\zeta$-function regularization and boundary conditions in one-loop quantum cosmology. I am also grateful to P. D'Eath for correspondence and conversations on quantum supergravity.

\section{References}

[1] G. Esposito, Quantum gravity, quantum cosmology and Lorentzian geometries, Lecture Notes in Physics, new series m: Monographs, vol. m12, second corrected and enlarged edition (Springer-Verlag, Berlin, 1994). 
[2] G. Esposito, G. Gionti, A.Yu. Kamenshchik, I.V. Mishakov and G. Pollifrone, Int. J. Mod. Phys. D 4 (1995) 735.

[3] P. D. D'Eath, Supersymmetric quantum cosmology (Cambridge University Press, Cambridge, 1996).

[4] G. Esposito and A.Yu. Kamenshchik, Phys. Rev. D 54 (1996) (hep-th 9604182).

[5] P.D. D'Eath and G. Esposito, Phys. Rev. D 44 (1991) 1713.

[6] H.C. Luckock and I.G. Moss, Class. Quantum Grav. 6 (1989) 1993.

[7] H.C. Luckock, J. Math. Phys. 32 (1991) 1755.

[8] I.G. Moss and S. Poletti, Nucl. Phys. B 341 (1990) 155.

[9] G. Esposito, A.Yu. Kamenshchik, I.V. Mishakov and G. Pollifrone, Phys. Rev. D 50 (1994) 6329.

[10] G. Esposito, A.Yu. Kamenshchik, I.V. Mishakov and G. Pollifrone, Phys. Rev. D 52 (1995) 3457.

[11] P.D. D'Eath, Phys. Rev. D 29 (1984) 2199.

[12] A.O. Barvinsky, A.Yu. Kamenshchik and I.P. Karmazin, Ann. Phys. (N.Y.) 219 (1992) 201.

[13] R. Endo, Class. Quantum Grav. 12 (1995) 1157.

[14] P.B. Gilkey, Invariance theory, the heat equation, and the Atiyah-Singer index theorem (Chemical Rubber Company, Boca Raton, 1995).

[15] A.O. Barvinsky, Phys. Lett. B 195 (1987) 344.

[16] I.G. Avramidi, G. Esposito and A.Yu. Kamenshchik, Class. Quantum Grav. 13 (1996) 2361. 